\title{
NOTE ON ASYMPTOTIC PRIME DIVISORS, ANALYTIC SPREADS AND THE ALTITUDE FORMULA
}

\author{
L. J. RATLIFF, JR. ${ }^{1}$
}

\begin{abstract}
The main result gives several characterizations of Noetherian domains that satisfy the altitude formula in terms of analytic spreads and asymptotic prime divisors.
\end{abstract}

1. Introduction. In [7], I proved several results concerning the prime divisors (= associated primes) of $I^{n}$ and the integral closure of $I^{n}$, where $I$ is an ideal in a Noetherian ring $R$ and $n$ is large. These results were considerably sharpened in [1] and [2]. In particular, in [1], Brodmann showed that all large powers of $I^{n}$ have the same prime divisors, and in [7, Theorem 2.5], I showed that the integral closures of all large powers of $I^{n}$ have the same prime divisors. Using this last result, McAdam proved, in [3, Theorem 3], the very interesting result that if $I \subseteq P$ are ideals in a Noetherian domain $R$ that satisfies the altitude formula and if $P$ is prime, then $P$ is a prime divisor of the integral closures of all large powers of $I^{n}$ if and only if (height $P$ ) equals the analytic spread of $I R_{P}$. Our main results, Theorems 1 and 2 , were suggested by McAdam's result and the related result [6, Theorem 2.29], and they characterize Noetherian domains that satisfy the altitude formula in terms of these asymptotic prime divisors and analytic spreads. Then, after proving several corollaries of these theorems, we close by showing certain additional rings (besides those in [3, Theorem 6]) have the property that a prime ideal is a prime divisor of all large powers of an ideal $I$ if and only if it is a prime divisor of the integral closures of all large powers of $I$.

2. Main results. Before proving our first characterization of the altitude formula, we first fix some notation and give the relevant definitions.

An integral domain $R$ satisfies the altitude formula if for all finitely generated extension domains $A$ of $R$ and all prime ideals $P$ in $A$, height $P+$ $\operatorname{trd}(A / P) /(R / p)=$ height $p+\operatorname{trd} A / R$, where $p=P \cap R$ and $\operatorname{trd} C / D$ denotes the transcendence degree of the integral domain $C$ over its subdomain $D$.

For an ideal $I$ in a Noetherian ring $R, v(I)$ denotes the minimum number of generators of $I$, and $I_{a}$ denotes the integral closure of $I$ in $R$ (so $I_{a}=\{x \in R ; x$ is a root of a polynomial of the form $T^{n}+i_{1} T^{n-1}+\cdots+i_{n}$, where $\left.\left.i_{j} \in I^{j}\right\}\right)$. $I$ is

Received by the editors June 25, 1980.

AMS (MOS) subject classifications (1970). Primary 13A15, 13C15; Secondary 13B25, $13 \mathrm{H} 10$.

Key words and phrases. Altitude formula, analytic spread of ideal, associated prime, height unmixed ideal, integral closure of ideal, local domain, Noetherian ring, regular local ring, system of parameters.

${ }^{1}$ Research on this paper was supported in part by the National Science Foundation, Grant MCS 77-00951-A02. 
height unmixed in case all prime divisors of $I$ have the same height. It is known [1] that all large powers of $I$ have the same prime divisors, and we let $A^{*}(I)$ denote this set: $A^{*}(I)=\operatorname{Ass}\left(R / I^{n}\right)$ for large $n$. Likewise, for all large $n$ the ideals $\left(I^{n}\right)_{a}$ have the same prime divisors, by [7, Theorem 2.5], and we let $\hat{A}^{*}(I)$ denote this set: $\hat{A}^{*}(I)=\operatorname{Ass}\left(R /\left(I^{n}\right)_{a}\right)$ for large $n$. Finally, $\Re(R, I)=R[t I, u]$ ( $t$ an indeterminate and $u=1 / t)$ is the Rees ring of $R$ with respect to $I$, and if $R$ is local with maximal ideal $M$, then the analytic spread $\mathfrak{I}(I)$ of $I$ is defined by $\mathfrak{I}(I)=$ altitude $\Re(R, I) /(u, M) \Re(R, I)$; hence $\mathfrak{I}(I)<$ altitude $R$.

For use in the proof of Theorem 1 , we note that $\Re=\Re(R, I)$ is a graded subring of $R[t, u]$ and $\Re\left(R_{S}, I R_{S}\right)=\Re_{S}$, if $S$ is an m.c. set in $R$ such that $I \cap S=\varnothing$. Also, if $R$ is local, then altitude $\Re=$ altitude $R+1$.

The following theorem shows more clearly the relationships between the conditions considered in [3, Theorem 3]. In the process of proving the theorem, we give a slightly different proof of [3, Theorem 3$]$.

THEOREM 1. The following statements are equivalent for a Noetherian domain $R$ :

(1.1) $R$ satisfies the altitude formula.

(1.2) If $I$ is an ideal in $R$ and $P \in \operatorname{Spec} R$ is a prime divisor of $\left(I^{n}\right)_{a}$ for some $n>1$, then height $P=\mathfrak{I}\left(I R_{P}\right)$.

(1.3) If $I \subseteq P$ are ideals in $R$ such that $P \in \hat{A}^{*}(I)$, then height $P=\mathfrak{I}\left(I R_{P}\right)$.

(1.4) If $I$ is an ideal in $R$ such that height $I=v(I)$, then $\left(I^{n}\right)_{a}$ is height unmixed for all $n \geqslant 1$.

Moreover, these statements imply, but are not equivalent to, the converse of (1.3).

Proof. It is clear that we may restrict attention to the case $I \neq(0)$.

Assume (1.1) holds, let $I$ be an ideal in $R$, and let $\Re=R[t I, u]$ be the Rees ring of $R$ with respect to $I$. Then it is shown in [7, Theorem 2.5] that if $P$ is a prime ideal in $R$ that is a prime divisor of $\left(I^{n}\right)_{a}$ for some $n$, then there exists a prime divisor $Q$ of $(u \Re)_{a}$ such that $Q \cap R=P$. Since $(1.1)$ holds, height $Q+\operatorname{trd}(\Re / Q) /(R / P)=$ height $P+\operatorname{trd} \Re / R$ and height $Q=1$, since $Q=Q^{\prime} \cap \Re$ with $Q^{\prime}$ a (height one) prime divisor of $u \Re^{\prime}$, where $\mathfrak{R}^{\prime}$ is the integral closure of $\Re$. Thus height $P=$ $\operatorname{trd}(\Re / Q) /(R / P)=\left(\right.$ say) $t$. Let $S=R-P$. Then altitude $\Re_{S}-1=$ altitude $R_{P}=$ height $P=t=\operatorname{trd}\left(\Re_{S} / Q \Re_{S}\right) /\left(R_{S} / P R_{S}\right)=[4, \quad(14.6)]$ altitude $\Re_{s} / Q \Re_{s}=\operatorname{depth} Q \Re_{s}$. Also, it is clear that depth $(u, P) \Re_{s}<$ altitude $\Re_{S}$ - $1=\operatorname{depth} Q \Re_{S}$ (as just shown), and $\operatorname{depth} Q \Re_{s}<\operatorname{depth}(u, P) \Re_{S} \quad$ (since $\left.(u, P) \Re_{S} \subseteq Q \Re_{S}\right)$, so height $P=t=\operatorname{depth} Q \Re_{S}=\operatorname{depth}(u, P) \Re_{S}=$ altitude $\Re_{S} /(u, P) \Re_{S}=\mathfrak{I}\left(I R_{P}\right)$. Therefore $(1.1) \Rightarrow(1.2)$.

It is clear that $(1.2) \Rightarrow(1.3)$.

Assume that (1.3) holds and that $I$ is an ideal in $R$ such that height $I=v(I)$. Fix $n \geqslant 1$ and let $P$ be a prime divisor of $\left(I^{n}\right)_{a}$. Then $P \in \hat{A}^{*}(I)$ by [7, Theorem 2.5] so (1.3) implies height $P=\mathfrak{l}\left(I R_{P}\right)$. Now it is clear that $\mathfrak{l}\left(I R_{P}\right)<v\left(I R_{P}\right)<v(I)=$ height $I<$ height $P$, so it follows that height $P=$ height $I$, and so (1.4) holds.

(1.4) $\Rightarrow$ (1.1) by [6, Theorem 2.29].

Next, assume (1.1) holds and let $I \subseteq P$ be ideals in $R$ such that $P$ is prime and height $P=\mathfrak{I}\left(I R_{P}\right)$. Then to show that $P \in \hat{A}^{*}(I)$ we may assume that $R$ is local 
and $P$ is its maximal ideal. Then $\mathfrak{I}\left(I R_{P}\right)=$ height $P$ implies $\mathfrak{I}(I)=$ altitude $R$, so altitude $\Re /(u, P) \Re=$ altitude $R$ where $\Re=\Re(R, I)$. Therefore height $(u, P) \Re=$ 1 , since $\operatorname{depth}(u, P) \Re=$ altitude $R$ and altitude $\Re=$ altitude $R+1$. Thus there exists a height one prime divisor $Q$ of $u \Re$ such that $Q \cap R=P$. Then $Q$ is a prime divisor of $(u \Re)_{a}$ so the proof of [7, Theorem 2.5] shows that $P$ is a prime divisor of $\left(I^{n}\right)_{a}$ for all large $n$. Therefore each of (1.1)-(1.4) implies the converse of (1.3).

Finally, let $R$ be a local domain of altitude two that is analytically unramified and whose integral closure has a height one maximal ideal. (For example, [4, pp. 203-205, Ex. 2] in the case $m=0$ and $r=1$.) Then $R$ does not satisfy the altitude formula. However, we now show that the converse of (1.3) does hold for $R$. Namely, let $I \subseteq P$ be ideals in $R$ such that $P$ is prime. If height $P=1$, then clearly height $P=\mathfrak{I}\left(I R_{P}\right)$ and $P \in \hat{A}^{*}(I)$. If height $P=2$, then $P$ is a prime divisor of all nonzero ideals contained in large powers of $P$ by $[8,(9)]$. Also, $R$ is analytically unramified, so $I^{n} \subseteq\left(I^{n}\right)_{a} \subseteq\left(P^{n}\right)_{a} \subseteq P^{m(n)}$ where $m(n) \rightarrow \infty$ with $n$, by [9, Theorem 2]. Therefore $P \in \hat{A}^{*}(I)$ for all nonzero ideals $I$ in $R$, so this holds, in particular when height $P=\mathfrak{I}\left(I R_{P}\right)$. Q.E.D.

Theorem 2 gives some additional characterizations of the altitude formula.

THEOREM 2. The following statements are equivalent for a Noetherian domain $R$ :

(2.1) $R$ satisfies the altitude formula.

(2.2) If $I \subseteq M$ are ideals in $R$ such that $M$ is maximal, height $I=v(I)$, and height $M / I=1$, then, for all $n \geqslant 1, M$ is not a prime divisor of $\left(I^{n}\right)_{a}$.

(2.3) If $I \subseteq M$ are ideals in $R$ such that $M$ is maximal, height $M / I=1$, and $I$ is integrally dependent on an ideal $B$ such that $v(B)=$ height $B$ then, for all $n>1, M$ is not a prime divisor of $\left(I^{n}\right)_{a}$.

(2.4) If $I$ is an ideal in $R$ that is generated by $h$ elements then, for all $n>1,\left(I^{n}\right)_{a}$ has no prime divisor of height $>h$.

Proof. (2.1) $\Leftrightarrow$ (2.2) by [6, Theorem 2.29].

It is clear that (2.3) $\Rightarrow(2.2)$. For the converse, let $B \subseteq I \subseteq M$ be as in (2.3). Then height $M / B=1$, so (2.2) implies $M$ is not a prime divisor of $\left(B^{n}\right)_{a}$ for all $n>1$. Since $B \subseteq I \subseteq B_{a}$, we have $\left(I^{n}\right)_{a}=\left(B^{n}\right)_{a}$ for all $n$, hence (2.2) $\Rightarrow(2.3)$.

$(2.4) \Rightarrow(2.1)$, by $(1.4) \Rightarrow(1.1)$. And, if (2.1) holds, if $I$ is as in (2.4), and if $P$ is a prime divisor of $\left(I^{n}\right)_{a}$ for some $n>1$, then height $P=\mathfrak{I}\left(I R_{P}\right)$, by $(1.1) \Rightarrow(1.2)$, and $\mathfrak{I}\left(I R_{P}\right) \leqslant v\left(I R_{P}\right) \leqslant v(I) \leqslant h$, so $(2.1) \Rightarrow(2.4)$. Q.E.D.

Remarks. (3.1) It is shown in [7, Theorem 2.5] that if a prime ideal $P$ in $R$ is a prime divisor of $\left(I^{n}\right)_{a}$ for some $n>1$, then $P$ is a prime divisor of $\left(I^{n}\right)_{a}$ for all large $n$. Therefore, the phrase "for all $n \geqslant 1$ " in (1.4), (2.2), and (2.3) can be replaced by "for infinitely many $n$ ".

(3.2) It is shown in [6, Example 2.28(b)] that there exists a local ring $(R, M)$ and an ideal $I$ in $R$ such that $v(I)=$ height $I=$ altitude $R-1, M \notin \hat{A}^{*}(I)$, and $R$ is not quasi-unmixed (which is equivalent to the altitude formula in the domain case). I do not know if this can hold when $R$ is an integral domain.

We will now give several corollaries of Theorems 1 and 2. 
COROLlary 4. Let $R$ be a Noetherian domain that satisfies the altitude formula and let $I \subseteq P$ be ideals in $R$ such that $P$ is prime. Then the following statements hold:

(4.1) [3, Theorem 3]. $P \in \hat{A}^{*}(I)$ if and only if height $P=\mathfrak{I}\left(I R_{P}\right)$.

(4.2) If $R$ is local with maximal ideal $M$ and if depth $I=1$, then height $I=\mathfrak{I}(I)$ = altitude $R-1$ if and only if $M \notin \hat{A}^{*}(I)$.

Proof. (4.1) is clear by Theorem 1 .

(4.2) Since depth $I=1$ and $R$ is catenary, height $I=$ altitude $R-1$. So, since $\mathfrak{I}(I)=\mathfrak{I}\left(I R_{M}\right),(4.2)$ readily follows from (4.1). Q.E.D.

REMARK 5. Concerning the condition height $I=\mathfrak{I}(I)$ in (4.2) (and also in (9.2), (9.3), and Corollary 11 ), note that if $R / M$ is infinite, then height $I=\mathfrak{I}(I)$ if and only if $I$ is integrally dependent on an ideal generated by height $I$ elements. For, if $R / M$ is infinite, then $\mathfrak{I}(I)$ is the number of elements in a minimal basis of a minimal reduction of $I$, by [5, Theorem 1 p. 150]. Therefore, if height $I=\mathfrak{I}(I)$, then $I$ is integrally dependent on an ideal generated by height $I$ elements. And, conversely, if $I$ is integrally dependent on such an ideal, then $I(I)<$ height $I$, by [5, Corollary, p. 151], and so height $I=\mathfrak{l}(I)$, by [5, Lemma 4, p. 151].

The next corollary is closely related to (4.2), and it shows, in particular, the well known fact that local domains of altitude one satisfy the altitude formula.

COROllary 6. Let $(R, M)$ be a local domain of altitude $d>1$. Then $R$ satisfies the altitude formula if and only if there does not exist a system of parameters $b_{1}, \ldots, b_{d}$ in $R$ such that $M$ is a prime divisor of $\left(\left(b_{1}, \ldots, b_{d-1}\right)^{n}\right)_{a}$ for some $n>1$.

Proof. If $R$ satisfies the altitude formula and $b_{1}, \ldots, b_{d}$ is a system of parameters in $R$, then height $\left(b_{1}, \ldots, b_{d-1}\right) R=d-1$ (since $R$ is catenary), so $M$ is not a prime divisor of $\left(\left(b_{1}, \ldots, b_{d-1}\right)^{n}\right)_{a}$ for all $n>1$, by $(2.1) \Rightarrow(2.2)$. Conversely, assume $R$ does not satisfy the altitude formula. Then, by (2.2) $\Rightarrow(2.1)$, there exists an ideal $I$ in $R$ such that depth $I=1, v(I)=$ height $I$, and $M$ is a prime divisor of $\left(I^{n}\right)_{a}$ for some $n$. Assume height $I=h$, let $\left(b_{1}, \ldots, b_{h}\right)$ be a minimal basis of $I$, and let $b_{h+1}$ in $M$ such that $b_{h+1}$ is not in any minimal prime divisor of $I$. Then $b_{1}, \ldots, b_{h+1}$ is a system of parameters in $R$, since depth $I=1$, so $h=d-1$ and the proof is complete.

The next corollary shows an interesting result for local domains that satisfy the altitude formula. It is, essentially, a special case of Corollary 6, and is analogous to the fact that the ideal $I$ is prime when $R$ is a Macaulay integral domain.

COROllary 7. Let $(R, M)$ be a local domain that satisfies the altitude formula, let altitude $R=d>1$, and let $L=R\left[X_{1}, \ldots, X_{h}\right]$, where $1<h<d$. Let $b_{0}, b_{1}, \ldots, b_{h}$ be a subset of a system of parameters in $R$, and let $I=$ $\left(b_{0} X_{1}-b_{1}, \ldots, b_{0} X_{h}-b_{h}\right) L$. Then $I_{a}$ is a prime ideal, $L_{I_{a}}$ is a regular local ring of altitude $h$, and $\left(I^{n}\right)_{a}$ is $I_{a}$-primary for all $n \geqslant 1$.

Proof. $L$ satisfies the altitude formula, since $R$ does, so if $P$ is a prime divisor of $\left(I^{n}\right)_{a}$ for some $n \geqslant 1$, then height $P=\mathfrak{l}\left(I L_{P}\right) \leqslant h$, by (1.1) $\Rightarrow(1.2)$. Also, $P \cap R=$ (0) and height $P=h$. To see this, note that $b_{0} \notin P \cap R$, since otherwise $b_{i}=b_{0} X_{i}$ $-\left(b_{0} X_{i}-b_{i}\right) \in P \cap R(i=1, \ldots, h)$, so $h \geqslant$ height $P \geqslant \operatorname{height}\left(b_{0}, \ldots, b_{h}\right) L=$ height $\left(b_{0}, \ldots, b_{h}\right) R=h+1$ (since $R$ is catenary) and this does not hold. Thus, 
with $p=P \cap R$ and $D=R_{p}\left[X_{1}, \ldots, X_{h}\right], P D$ is proper and contains $Q=$ $\left(p R_{p}, X_{1}-\left(b_{1} / b_{0}\right), \ldots, X_{h}-\left(b_{h} / b_{0}\right)\right) D$, so $h>$ height $P D>$ height $Q=$ height $p R_{p}+h$, thus height $p R_{p}=0$, and so $p=P \cap R=(0)$ and height $P=h$. Therefore $R_{p}$ is the quotient field $F$ of $R$ and $P D=Q$, so $L_{P}=D_{Q}=$ $F\left[X_{1}, \ldots, X_{h}\right]_{Q}$ is a regular local ring of altitude $h$. Also, it follows that $\left(I L_{P}\right)_{a}=$ $I_{a} L_{P}=Q D_{Q}=I L_{P}$ (since $I_{a} R_{S}=\left(I R_{S}\right)_{a}$ always holds and since $I L_{P}$ is prime). Hence, since none of the ideals $\left(I^{n}\right)_{a}$ has a prime divisor of height $>h$, it follows that $I_{a}$ is prime and $\left(I^{n}\right)_{a}$ is $I_{a}$-primary. Q.E.D.

REMARK 8. It would be interesting to know if the following strong converse of Corollary 7 holds: if there exists a system of parameters $b_{1}, \ldots, b_{d}$ in $R$ such that the conclusion of Corollary 7 holds, then $R$ satisfies the altitude formula.

(9.1) is a small generalization of (4.2), and (9.2) and (9.3) contain some additional information. In the proofs of (9.2) and (9.3) we use $\mathfrak{I}\left(I R_{P}\right)<\mathfrak{I}(I)$. I do not know a reference for this, but it is fairly straightforward to prove, and it holds in all local rings $R$ and for all ideals $I \subseteq P$ with $P$ prime.

COROllary 9. Let $(R, M)$ be a local domain of altitude $d$ that satisfies the altitude formula and let $I$ be an ideal in $R$ such that height $I<d$. Then the following statements hold:

(9.1) $M \notin \hat{A}^{*}(I)$ if and only if $\mathrm{I}(I)<d$.

(9.2) If there exists $P \in \hat{A}^{*}(I)$ such that height $I=$ height $P$ and $\mathfrak{I}(I)=\mathfrak{I}\left(I R_{P}\right)$, then height $I=\mathfrak{I}(I)$.

(9.3) If height $I=\mathfrak{l}(I)$, then $\left(I^{n}\right)_{a}$ is height unmixed for all $n \geqslant 1$.

Proof. (9.1) By (4.1), $M \notin \hat{A}^{*}(I)$ if and only if height $M \neq \mathfrak{I}\left(I R_{M}\right)=\mathfrak{I}(I)$, so if and only if $\mathrm{I}(I)<$ height $M=d$.

(9.2) If there exists such $P \in \hat{A}^{*}(I)$, then height $I=$ height $P=$ (by (4.1)) $\mathfrak{l}\left(I R_{P}\right)<\mathfrak{l}(I)=$ height $I$, so height $I=\mathfrak{l}(I)$.

(9.3) Let $P \in \hat{A}^{*}(I)$. Then $\mathfrak{I}(I)=$ height $I<$ height $P=$ (by (4.1)) $\mathfrak{I}\left(I R_{P}\right)<$ I(I), so height $P=$ height $I$. Q.E.D.

REMARK 10. It follows readily from $(9.3)$, and $(1.4) \Rightarrow(1.1)$ together with [5, Lemma 4, p. 151], that if $(R, M)$ is a local domain such that $R / M$ is infinite, then $R$ satisfies the altitude formula if and only if for all ideals $I$ in $R$ such that height $I=\mathfrak{I}(I),\left(I^{n}\right)_{a}$ is height unmixed for all $n>1$. And two additional characterizations similar to $(2.2)$ and (2.3) with $\mathfrak{I}$ replacing $v$ readily follow from this.

The next corollary is an important special case of (10.3), and is somewhat analogous to the fact that if $Q$ is a primary ideal in a Macaulay ring such that height $Q=v(Q)$, then $Q^{n}$ is primary for all $n$.

COROLlaRY 11. Let $R$ be as in Corollary 9 and let $Q$ be a primary ideal in $R$. If height $Q=\mathfrak{I}(Q)$, then $\left(Q^{n}\right)_{a}$ is primary for all $n>1$.

Proof. By (9.3), all the ideals $\left(Q^{n}\right)_{a}$ are height unmixed, so the conclusion follows, since $\operatorname{Rad}\left(Q^{n}\right)_{a}=\operatorname{Rad} Q$ is prime. Q.E.D.

This paper will be closed with the following proposition which is related to [3, Theorem 6]. (As noted at the start of this section, $A^{*}(I)=\operatorname{Ass}\left(R / I^{n}\right)$ for large $n$.) 
Proposition 12. Let $(R, M)$ be a local domain of altitude two or three such that there exists a height one maximal ideal in $R^{\prime}$, the integral closure of $R$. Assume $R$ is analytically unramified and $R_{P}$ is integrally closed for all nonmaximal height two prime ideals $P$ in $R$. Then $A^{*}(I)=\hat{A}^{*}(I)$ for all ideals $I$ in $R$.

Proof. Exactly as in the last paragraph of the proof of Theorem $1, M$ is in $\hat{A}^{*}(I)$ and in $A^{*}(I)$. If altitude $R=3$ and $P$ is a height two prime ideal in $R$, then $R_{P}$ is integrally closed, so [3, Theorem 6] shows that $P \in A^{*}(I)$ if and only if $P \in$ $\hat{A}^{*}(I)$. Finally, if height $P=1$, then clearly $P \in A^{*}(I)$ if and only if $P \in \hat{A}^{*}(I)$. Q.E.D.

It should be noted that Nagata's examples in the cases $m=0$ and $r=1$ or 2 satisfy the conditions on $R$ in Proposition 12.

\section{REFERENCES}

1. M. Brodmann, Asymptotic stability of Ass(M/In $M)$, Proc. Amer. Math. Soc. 74 (1979), 16-18.

2. P. Eakin and S. McAdam, The asymptotic Ass, J. Algebra 61 (1979), 71-81.

3. S. McAdam, Asymptotic prime divisors and analytic spreads, Proc. Amer. Math. Soc. 80 (1980), 555-559.

4. M. Nagata, Local rings, Interscience Tracts 13, Interscience, New York, 1962.

5. D. G. Northcott and D. Rees, Reductions of ideals in local rings, Proc. Cambridge Philos. Soc. 50 (1954), 145-158.

6. L. J. Ratliff, Jr., Locally quasi-unmixed Noetherian rings and ideals of the principal class, Pacific J. Math. 52 (1974), 185-205.

7. __ On prime divisors of $I^{n}, n$ large, Michigan Math. J. 23 (1976), 337-352.

8. 81 (1979), 537-545.

9. M. Sakuma and H. Okuyama, On a criterion for analytically unramification of a local ring, J. Gakugei Tokushima Univ. 15 (1966), 36-38.

Department of Mathematics, Universtty of California, Riverside, California 92521 